\title{
Brain anatomical variations among Malaysian population
}

\begin{abstract}
Spatial normalization is an important task in neuroimaging analysis to compensate with the anatomical variations between different subjects. Current practice is to normalize the sample image to the reference brain atlas to ensure that the unbiased image-to-image comparison is possible. The available template nowadays is ICBM152, which derived from a Caucasian population. Since the brain anatomical variations has been reported especially for the interregional cohorts, the use of the ICBM152 template for other region subjects is questionable. In addition to that, several other factors such as age and gender have also been reported to have an effect to the brain morphometric. This study investigates the global brain shape measures for different group among Asian population. It is useful as a basis for further investigation on a more complex brain structure differences among local and inter-regional population. Later on, a group-specific atlas may be constructed to be used exclusively for Asian subjects. This study showed that gender factor has a significant effect on the brain shape while the age and race demonstrated no significant correlation.
\end{abstract}

Keyword: Neuroimaging analysis; Brain anatomical variations; Spatial normalization; Malaysian population; Global brain shape measures; Asian population 